[5] A. V. Savkin and R. J. Evans, Hybrid Dynamical Systems - Controller and Sensor Switching Problems. Boston, MA: Birkhauser, 2002.

[6] J. P. Hespanha, "Uniform stability of switched linear systems: Extensions of LaSalle's invariance principle," IEEE Trans. Autom. Control, vol. 49, no. 4, pp. 470-482, Apr. 2004.

[7] P. Colaneri, "Dwell time analysis of deterministic and stochastic switched systems," Eur. J. Autom. Control, vol. 15, pp. 228-249, 2009.

[8] S. Boyarski and U. Shaked, "Time-convexity and time-gain-scheduling in finite-horizon robust $H_{\infty}$-control," in Proc. 48th CDC'09, Shanghai, China, 2009, pp. 2765-2770.

[9] G. Xie and L. Wang, "Stabilization of switched linear systems with time-delay in detection of switching signal," J. Math. Anal. Appl., vol. 305, pp. 277-290, 2005.

[10] S. Boyd, L. El Ghaoui, E. Feron, and V. Balakrishnan, Linear Matrix Inequalities in System and Control Theory. Philadelphia, PA: SIAM, 1994.

[11] P. Apkarian and P. Gahinet, "A convex characterization of gain-scheduled $H_{\infty}$ conterollers," IEEE Trans. Autom. Control, vol. 40, no. 5, pp. 853-864, May 1995.

[12] M. C. de Oliveira and R. E. Skelton, "Stability test for constrained linear systems," in Perspectives in Robust Control, S. O. Reza Moheimani, Ed. London, U.K.: Springer, 2001, vol. 268, Lecture Notes in Control and Information Sciences.

[13] J. C. Geromel, M. C. de Oliveira, and L. Hsu, "LMI characterization of structural and robust stability," Lin. Algebra Appl., vol. 285, pp. 69-80, 1998.

\section{On the Convergence Time of Asynchronous Distributed Quantized Averaging Algorithms}

\author{
Minghui Zhu and Sonia Martínez
}

\begin{abstract}
We introduce here a class of distributed quantized averaging algorithms for asynchronous communication networks with fixed and switching topologies. The focus of this technical note is on the study of the convergence time of the proposed quantized averaging algorithms. By appealing to random walks on graphs, we derive polynomial bounds on the expected convergence time of the algorithms presented, as a function of the number of agents in the network.
\end{abstract}

Index Terms-Consensus algorithms, cooperative control, multi-agent systems.

\section{INTRODUCTION}

The objective of the distributed consensus problem is the design of algorithms that allow a group of agents to reach agreement by communicating locally with neighbors. This problem historically roots in parallel computation [2], and has attracted significant attention recently [3], [11], [15]. More specifically, the objective of the distributed averaging problem is that each agent reaches consensus on the average of individual initial states.

In ad hoc sensor and communication networks, the capacities of communication channels and the memory functions of nodes are finite.

Manuscript received October 16, 2008; revised May 29, 2009 and February 12, 2010; accepted September 14, 2010. Date of publication November 18, 2010; date of current version February 09, 2011. This work is supported by the NSF CAREER Award CMS-0643673 and NSF IIS-0712746. Recommended by Associate Editor Z. Qu.

The authors are with the Department of Mechanical and Aerospace Engineering, University of California at San Diego, La Jolla CA, 92093 USA (e-mail: mizhu@ucsd.edu; soniamd@ucsd.edu).

Digital Object Identifier 10.1109/TAC.2010.2093276
Furthermore, computations can only be carried out with finite precision. From a practical point of view, real-valued averaging algorithms are not feasible and these realistic constraints motivate the problem of average consensus via quantized information. Another motivation for distributed quantized averaging is load balancing with indivisible tasks. Prior work on distributed quantized averaging over fixed graphs includes [1], [6], [7], [12]. Recently, [14] examines quantization effects on distributed averaging algorithms over time-varying topologies. As in [12], we focus on gossip-based quantized averaging algorithms preserving the sum of the state values at each iteration. This setup has the following properties of interest: the sum cannot be changed in some situations, such as load balancing; and the constant sum leads to a small steady-state error with respect to the average of individual initial states. This error is equal to one quantization step size or zero (when the average of the initial states is located at one of the quantization levels) and thus is independent of $N$.

The worst-case upper bound on the convergence time-as a function of $N$ and independent of network topologies - is a typical measure to quantify the performance of real-valued averaging (e.g., in [16]) and quantized averaging (e.g., in [12], [14]). In particular, polynomial bounds over fixed complete and linear graphs are derived in [12]; and, the authors in [14] give a polynomial bound over switching topologies. To explicitly find these bounds, the paper [14] requires a common time-slotted system, and [12] needs some global information (e.g., a centralized entity choosing one edge to establish communication or the number of the edges available in the graph). However, real-world communication networks are inherently asynchronous ${ }^{1}$ environment and lack of centralized coordination. These constraints motivate us to employ the asynchronous network model in [4] to select one edge at each time instant in a totally distributed manner without any global information.

After the submission of this work, the manuscripts [9], [13] have focused on gossip-based quantized averaging algorithms and characterized the bounds on their convergence rates for fixed and connected graphs. Instead of being functions of $N$, these bounds depend on graph topologies. Our results, developed independently from [9], [13], provide general bounds on the expected convergence time over fixed and switching topologies.

Statement of Contributions: The present technical note proposes a class of distributed quantized averaging algorithms on asynchronous communication networks with fixed and switching topologies. The algorithms are shown to asymptotically reach quantized average consensus almost surely. Furthermore, we utilize meeting times of two random walks on graphs as a unified approach to derive polynomial bounds on their expected convergence times. To the best of our knowledge, this note derives for the first time such polynomial bounds without requiring any global information. We refer the readers to [18] for an enlarged version of this note that includes all the proofs. A preliminary conference version of this technical note is in [17] where the convergence time of synchronous algorithms is also studied.

\section{Preliminaries And Problem Statement}

Here, we present the problem formulation along with some notation and terminology.

Asynchronous Time Model: In this note, we will employ the asynchronous time model proposed in [4]. More precisely, consider a network of $N$ nodes, labeled 1 through $N$. Each node has a clock which ticks according to a rate 1 Poisson process. Hence, the inter-tick

\footnotetext{
${ }^{1}$ As in [4], asynchronism in this note means that time is not assumed to be slotted commonly across nodes.
} 
times at each node are random variables with rate 1 exponential distribution, independent across nodes and independent over time. By the superposition theorem for Poisson processes, this setup is equivalent to a single global clock modeled as a rate $N$ Poisson process ticking at times $\left\{Z_{k}\right\}_{k>0}$. By the orderliness property of Poisson processes, the clock ticks do not occur simultaneously. The inter-agent communication and the update of consensus states only occur at $\left\{Z_{k}\right\}_{k>0}$. In the reminder of this technical note, the time instant $t$ will be discretized according to $\left\{Z_{k}\right\}_{k>0}$ and defined in terms of the number of clock ticks.

Network Model: We will employ the undirected graph $\mathcal{G}(t)=$ $(V, E(t))$ to model the network. Here $V:=\{1, \cdots, N\}$ is the vertex set, and an edge $(j, i) \in E(t)$ if and only if node $j$ can receive the message from node $i$ (e.g., node $j$ is within the communication range of node $i$ ) at time $t$. The neighbors of node $i$ at time $t$ are denoted by $\mathcal{N}_{i}(t)=\{j \in V \mid(j, i) \in E(t)$ and $j \neq i\}$. The state of node $i$ at time $t$ is denoted by $x_{i}(t) \in \mathbb{R}$ and the network state is denoted by $x(t)=\left(x_{1}(t), \cdots, x_{N}(t)\right)^{T}$. Suppose the initial states $x_{i}(0) \in\left[U_{\min }, U_{\max }\right]$ for all $i \in V$ and some real numbers $U_{\min }$ and $U_{\max }$.

Quantization Scheme: Let $R$ denote the number of bits per sample. The total number of quantization levels can be represented by $L=2^{R}$ and the step size is $\Delta=\left(U_{\max }-U_{\min }\right) / 2^{R}$. The set of quantization levels, $\left\{\omega_{1}, \cdots, \omega_{L}\right\}$, is a strictly increasing sequence in $\mathbb{R}$ and the levels are uniformly spaced in the sense that $\omega_{i+1}-\omega_{i}=\Delta$. A quantizer $\mathcal{Q}:\left[U_{\min }, U_{\max }\right] \rightarrow\left\{\omega_{1}, \cdots, \omega_{L}\right\}$ is adopted to quantize the message $u \in\left[U_{\min }, U_{\max }\right]$ in such a way that $\mathcal{Q}(u)=\omega_{i}$ if $u \in\left[\omega_{i}, \omega_{i+1}\right)$ for some $i \in\{1, \cdots, L-1\}$. Assume that the initial states $x_{i}(0)$ for all $i \in V$ are multiples of $\Delta$.

Problem Statement: The problem of interest in this technical note is to design distributed averaging algorithms which the nodes can utilize to update their states by communicating with neighbors via quantized messages in an asynchronous setting. Ultimately, quantized average consensus is reached in probability; i.e., for any initial state $x(0)$, there holds that $\lim _{t \rightarrow \infty} \mathbb{P}(x(t) \in \mathcal{W}(x(0)))=1$. The set $\mathcal{W}(x(0))$ is dependent on initial state $x(0) \in \mathbb{R}^{N}$ and defined as follows. If $\bar{x}(0)=$ $(1 / N) \sum_{i=1}^{N} x_{i}(0)$ is not a multiple of $\Delta$, then $\mathcal{W}(x(0))=\{x \in$ $\left.\mathbb{R}^{N} \mid x_{i} \in\{\mathcal{Q}(\bar{x}(0)), \mathcal{Q}(\bar{x}(0))+\Delta\}\right\} ;$ otherwise, $\mathcal{W}(x(0))=\{x \in$ $\left.\mathbb{R}^{N} \mid x_{i}=\bar{x}(0)\right\}$. Now it is clear that the steady-state error is at most $\Delta$ after quantized average consensus is reached.

Notions of Random Walks on Graphs: In this technical note, random walks on graphs play an important role in characterizing the convergence properties of our quantized averaging algorithms. The following definitions are generalized from those defined for fixed graphs in [5], [8].

Definition 2.1 (Random Walks): A random walk on the graph $\mathcal{G}(t)$ under the transition matrix $P(t)=\left(p_{i j}(t)\right)$, starting from node $v$ at time $s$, is a stochastic process $\{X(t)\}_{t \geq s}$ such that $X(s)=v$ and $\mathbb{P}(X(t+1)=j \mid X(t)=i)=p_{i j}(t)$. A random walk is said to be simple if for any $i \in V, p_{i i}(t)=0$ for all $t \geq 0$; otherwise, it is said to be natural.

Definition 2.2 (Hitting Time): Consider a random walk on the graph $\mathcal{G}(t)$, beginning from node $i$ at time $s$ and evolving under the transition matrix $P(t)$. The hitting time from node $i$ to the set $\Lambda \subseteq V$, denoted as $H_{(\mathcal{G}(t), P(t), s)}(i, \Lambda)$, is the expected time it takes this random walk to reach the set $\Lambda$ for the first time. We denote $H_{(\mathcal{G}(t), P(t))}(\Lambda)=$ $\sup _{s>0} \max _{i \in V} H_{(\mathcal{G}(t), P(t), s)}(i, \Lambda)$ as the hitting time to reach the set $\Lambda$. The hitting time of the pair $i, j$, denoted as $H_{(\mathcal{G}(t), P(t), s)}(i, j)$, is the expected time it takes this random walk to reach node $j$ for the first time. Denote $H_{(\mathcal{G}(t), P(t))}=\sup _{s \geq 0} \max _{i, j \in V} H_{(\mathcal{G}(t), P(t), s)}(i, j)$ as the hitting time of going between any pair of nodes.
Definition 2.3 (Meeting Time): Consider two random walks on the graph $\mathcal{G}(t)$ under the transition matrix $P(t)$, starting at time $s$ from node $i$ and node $j$ respectively. The meeting time $M_{(\mathcal{G}(t), P(t), s)}(i, j)$ of these two random walks is the expected time it takes them to meet at some node for the first time. The meeting time on the graph $\mathcal{G}(t)$ is defined as $M_{(\mathcal{G}(t), P(t))}=\sup _{s \geq 0} \max _{i, j \in V} M_{(\mathcal{G}(t), P(t), s)}(i, j)$.

For the ease of notation, we will drop the subscript $s$ in the hitting time and meeting time notions for fixed graphs.

Notations: For $\alpha \in \mathbb{R}$, define $V_{\alpha}: \mathbb{R}^{N} \rightarrow \mathbb{R}$ as $V_{\alpha}(x)=\sum_{i=1}^{N}\left(x_{i}-\alpha\right)^{2}$. We define $J: \mathbb{R}^{N} \rightarrow \mathbb{R}$ as $J(x)=$ $\left(\max _{i \in V} x_{i}-\min _{i \in V} x_{i}\right) / \Delta$. Denote the set $\Theta=\{(k, k) \mid k \in V\}$. The distribution of a vector $x \in \mathbb{R}^{N}$ is defined to be the list $\left\{\left(q_{1}, m_{1}\right),\left(q_{2}, m_{2}\right), \cdots,\left(q_{k}, m_{k}\right)\right\}$ for some $k \in V$ where $\sum_{\ell=1}^{k} m_{\ell}=N, q_{i} \neq q_{j}$ for $i \neq j$ and $m_{\ell}$ is the cardinality of the set $\left\{i \in V \mid x_{i}=q_{\ell}\right\}$. The cardinality of the set $M$ is denoted by $|M|$.

\section{ASYNCHRONOUS DISTRIBUTED QUANTIZED AVERAGING ON FIXED GRAPHS}

In this section, we propose and analyze an asynchronous distributed quantized averaging algorithm on the fixed and connected graph $\mathcal{G}$. Main references are [12] on quantized gossip algorithms and [5] on the meeting time of two simple random walks on fixed graphs.

\section{A. Proposed Algorithm}

The asynchronous distributed quantized averaging algorithm on the fixed and connected graph $\mathcal{G}$ (AF, for short) is described as follows. Suppose node $i$ 's clock ticks at time $t$. Node $i$ randomly chooses one of its neighbors, say node $j$, with equal probability. Node $i$ and $j$ then execute the following local computation. If $x_{i}(t) \geq x_{j}(t)$, then

$$
x_{i}(t+1)=x_{i}(t)-\delta, \quad x_{j}(t+1)=x_{j}(t)+\delta
$$

otherwise

$$
x_{i}(t+1)=x_{i}(t)+\delta, \quad x_{j}(t+1)=x_{j}(t)-\delta
$$

where $\delta=(1 / 2)\left(x_{i}(t)-x_{j}(t)\right)$ if $\left(x_{i}(t)-x_{j}(t)\right) /(2 \Delta)$ is an integer; otherwise, $\delta=\mathcal{Q}\left((1 / 2)\left(x_{i}(t)-x_{j}(t)\right)\right)+\Delta$. Every other node $k \in$ $V \backslash\{i, j\}$ preserves its current state; i.e., $x_{k}(t+1)=x_{k}(t)$.

Remark 3.1: The precision $\Delta / 2$ is sufficient for the computation of $\delta$ and thus the update laws (1) and (2). It is easy to verify that $x_{i}(t) \in$ $\left[U_{\min }, U_{\max }\right]$ and $x_{i}(t)$ are multiples of $\Delta$ for all $i \in V$ and $t \geq 0$. Furthermore, the sum of the state values is preserved at each iteration.

If $\left|x_{i}(t)-x_{j}(t)\right|=\Delta$, the update laws (1) and (2) become $x_{i}(t+$ $1)=x_{j}(t)$ and $x_{j}(t+1)=x_{i}(t)$. Such update is referred to as a trivial average in [12]. If $\left|x_{i}(t)-x_{j}(t)\right|>\Delta$, then (1) or (2) is referred to as a non-trivial average. Although it does not directly contribute to reaching quantized average consensus, trivial average helps the information flow over the network.

\section{B. The Meeting Time of Two Natural Random Walks on a Fixed Graph $\mathcal{G}$}

We first study a variation of the problem in [8], namely, the meeting time of two natural random walks on a fixed graph $\mathcal{G}$. More precisely, assume that the fixed graph $\mathcal{G}$ be undirected and connected. Initially, two tokens are placed on the graph $\mathcal{G}$; at each time, one of the tokens is chosen with probability $1 / N$ and the chosen token moves to one of the neighboring nodes with equal probability. What is the meeting time for these two tokens?

The tokens move as two natural random walks with the transition matrix $P_{\mathrm{AF}}$ on the graph $\mathcal{G}$. The matrix $P_{\mathrm{AF}}=\left(\tilde{p}_{i j}\right) \in \mathbb{R}^{N \times N}$ is given by $\tilde{p}_{i i}=1-(1 / N)$ for $i \in V, \tilde{p}_{i j}=1 /\left(N\left|\mathcal{N}_{i}\right|\right)$ for $(i, j) \in E$. Their meeting time is denoted as $M_{\left(\mathcal{G}, P_{\mathrm{AF}}\right)}$. Denote any of these two natural random walks as $X_{\mathcal{N}}$. Correspondingly, we construct a simple 
random walk, say $X_{\mathcal{S}}$, with the transition matrix $P_{\mathrm{SF}}$ on the graph $\mathcal{G}$ where $P_{\mathrm{SF}}=\left(p_{i j}\right) \in \mathbb{R}^{N \times N}$ is given by $p_{i i}=0$ and $p_{i j}=1 /\left|\mathcal{N}_{i}\right|$ if $(i, j) \in E$. The hitting times of $X_{\mathcal{S}}$ and $X_{\mathcal{N}}$ are denoted as $H_{\left(\mathcal{G}, P_{\mathrm{SF}}\right)}$ and $H_{\left(\mathcal{G}, P_{\mathrm{AF}}\right)}$, respectively.

Proposition 3.1: The meeting time of two natural random walks with transition matrix $P_{\mathrm{AF}}$ on the fixed graph $\mathcal{G}$ satisfies that $M_{\left(\mathcal{G}, P_{\mathrm{AF}}\right)} \leq 2 N H_{\left(\mathcal{G}, P_{\mathrm{SF}}\right)}-N$.

Proof: Since the fixed graph $\mathcal{G}$ is undirected and connected, the random walks $X_{\mathcal{N}}$ and $X_{\mathcal{S}}$ are irreducible. The proof is based on the following sequence of claims:

i) It holds that $H_{\left(\mathcal{G}, P_{\mathrm{AF}}\right)} \geq N$.

ii) For any pair $i, j \in V$ with $i \neq j$, we have $H_{\left(\mathcal{G}, P_{\mathrm{AF}}\right)}(i, j)=$ $N H_{\left(\mathcal{G}, P_{\mathrm{SF}}\right)}(i, j)$.

iii) For any $i, j, k \in V$, the following equality holds:

$$
\begin{aligned}
H_{\left(\mathcal{G}, P_{\mathrm{AF}}\right)}(i, j) & +H_{\left(\mathcal{G}, P_{\mathrm{AF}}\right)}(j, k)+H_{\left(\mathcal{G}, P_{\mathrm{AF}}\right)}(k, i) \\
& =H_{\left(\mathcal{G}, P_{\mathrm{AF}}\right)}(i, k)+H_{\left(\mathcal{G}, P_{\mathrm{AF}}\right)}(k, j)+H_{\left(\mathcal{G}, P_{\mathrm{AF}}\right)}(j, i) .
\end{aligned}
$$

iv) There holds that $M_{\left(\mathcal{G}, P_{\mathrm{AF}}\right)} \leq 2 H_{\left(\mathcal{G}, P_{\mathrm{AF}}\right)}-N$.

Due to the space limitation, we omit the details of the proofs. The interested reader can find a complete proof in [18]

\section{Convergence Analysis of AF}

We now proceed to analyze the convergence properties of AF. The convergence time of $\mathrm{AF}$ is a random variable defined as follows: $T_{\text {con }}(x(0))=\inf \{t \mid x(t) \in \mathcal{W}(x(0))\}$, where $x(t)$ starts from $x(0)$ and evolves under AF. Choose $V_{\bar{x}(0)}(x)=\sum_{i=1}^{N}\left(x_{i}-\bar{x}(0)\right)^{2}$ as a Lyapunov function candidate for AF. One can readily see that $V_{\bar{x}(0)}(x(t+1))=V_{\bar{x}(0)}(x(t))$ when a trivial average occurs and $V_{\bar{x}(0)}(x)$ reduces at least $2 \Delta^{2}$ when a non-trivial average occurs. Hence, $V_{\bar{x}(0)}(x)$ is non-increasing along the trajectories, and the number of non-trivial averages is at most $1 /\left(2 \Delta^{2}\right) V_{\bar{x}(0)}(x(0))$. Define the set $\Psi=\{x \in$ $\mathbb{R}^{N} \mid$ the distribution of $x$ is $\left.\{(0,1),(\Delta, N-2),(2 \Delta, 1)\}\right\}$ and denote $\mathbb{E}\left[T_{\Psi}\right]=\max _{x(0) \in \Psi} \mathbb{E}\left[T_{\text {con }}(x(0))\right]$. It is clear that the expected time between any two consecutive non-trivial averages is not larger than $\mathbb{E}\left[T_{\Psi}\right]$. Then we have the following estimates on $\mathbb{E}\left[T_{\text {con }}(x(0))\right]$ :

$$
\mathbb{E}\left[T_{\text {con }}(x(0))\right] \leq \frac{1}{2 \Delta^{2}} V_{\bar{x}(0)}(x(0)) \mathbb{E}\left[T_{\Psi}\right] \leq \frac{N J(x(0))^{2}}{8} \mathbb{E}\left[T_{\Psi}\right]
$$

where the second inequality is a direct result of Lemma 4 in [12].

Theorem 3.1: For any $x(0) \notin \mathcal{W}(x(0))$, the expected convergence time $\mathbb{E}\left[T_{\text {con }}(x(0))\right]$ of $\mathrm{AF}$ is upper bounded by $\left(N^{2} J(x(0))^{2} / 8\right)\left((8 / 27) N^{3}-1\right)$. Furthermore, almost any evolution $x(t)$ starting from $x(0)$ reaches quantized average consensus.

Proof: By (3), it suffices to bound $\mathbb{E}\left[T_{\Psi}\right]$. Assume that $x(0) \in \Psi$. Before they meet for the first time, the values 0 and $2 \Delta$ move as two natural random walks which are identical to $X_{\mathcal{N}}$ in Proposition 3.1. At their meeting for the first time, the values of 0 and $2 \Delta$ average and quantized average consensus is reached. Hence, $\mathbb{E}\left[T_{\Psi}\right]=M_{\left(\mathcal{G}, P_{\mathrm{AF}}\right)}$ and thus inequality (3) becomes

$$
\begin{aligned}
\mathbb{E}\left[T_{\text {con }}(x(0))\right] & \leq \frac{N J(x(0))^{2}}{8} M_{\left(\mathcal{G}, P_{\mathrm{AF}}\right)} \\
& \leq \frac{N J(x(0))^{2}}{8}\left(2 N H_{\left(\mathcal{G}, P_{\mathrm{SF}}\right)}-N\right)
\end{aligned}
$$

where we use Proposition 3.1 in the second inequality. By letting $M=0$ in the theorem of Page 265 in [5], we can obtain the upper bound $(4 / 27) N^{3}$ on $H_{\left(\mathcal{G}, P_{\mathrm{SF}}\right)}$. Substituting this upper bound into inequality (4) gives the desired upper bound on $\mathbb{E}\left[T_{\text {con }}(x(0))\right]$. The remainder of the proofs can be completed by using the property of the set $\mathcal{W}(x(0))$ being absorbing and Markov's inequality. We omit the details here.

\section{ASYNCHRONOUS DISTRIBUTED QUANTIZED AVERAGING ON SWITCHING GRAPHS}

We now turn our attention to the more challenging scenario where the communication graphs are undirected but dynamically changing. We will propose and analyze an asynchronous distributed quantized averaging algorithm on switching graphs (AS, for short). The convergence rate of distributed real-valued averaging algorithms on switching graphs in [14] will be employed to characterize the hitting time of random walks on switching graphs.

\section{A. Proposed Algorithm}

The main steps of AS can be summarized as follows. At time $t$, let node $i$ 's clock tick. If $\left|\mathcal{N}_{i}(t)\right| \neq 0$, node $i$ randomly chooses one of its neighbors, say node $j$, with probability $1 / \max \left\{\left|\mathcal{N}_{i}(t)\right|,\left|\mathcal{N}_{j}(t)\right|\right\}$. Then, node $i$ and $j$ execute the computation (1) or (2) and every other node $k \in V \backslash\{i, j\}$ preserves its current state. If $\left|\mathcal{N}_{i}(t)\right|=0$, all the nodes do nothing at this time.

Here, we assume that the communication graph $\mathcal{G}(t)$ be undirected and satisfies the following connectivity assumption also used in [3], [11], [14], [16].

Assumption 4.1 (Periodical Connectivity): There exists some $B \in$ $\mathbb{N}_{>0}$ such that, for all $t \geq 0$, the undirected graph $(V, E(t) \cup E(t+$ 1) $\cup \cdots \cup E(t+B-1))$ is connected.

Remark 4.1: In the AS, the probability that node $i$ chooses a neighbor $j$ is $1 / \max \left\{\left|\mathcal{N}_{i}(t)\right|,\left|\mathcal{N}_{j}(t)\right|\right\}$. Thus, this information should be available to node $i$. In this way, the matrix $P_{\mathrm{AS}}(t)$ defined later is symmetric and double stochastic.

\section{B. The Meeting Time of Two Natural Random Walks on a Time-Varying Graph $\mathcal{G}(t)$}

Before analyzing AS, we consider the following problem which generalizes the problem in Section III-B to the case of dynamically changing graphs.

The meeting time of two natural random walks on a time-varying graph $\mathcal{G}(t)$. Assume that $\mathcal{G}(t)$ be undirected and satisfies Assumption 4.1. Initially, two tokens are placed on $\mathcal{G}(0)$. At each time, one of the tokens is chosen with probability $1 / N$. The chosen token at some node, say $i$, moves to one of the neighbors, say node $j$, with probability $1 / \max \left\{\left|\mathcal{N}_{i}(t)\right|,\left|\mathcal{N}_{j}(t)\right|\right\}$ if $\left|\mathcal{N}_{i}(t)\right| \neq 0$; otherwise, it will stay up with node $i$. What is the meeting time for these two tokens?

Clearly, the movements of two tokens are two natural random walks, say $X_{1}$ and $X_{2}$, on the switching graph $\mathcal{G}(t)$. Their meeting time is denoted as $M_{\left(\mathcal{G}(t), P_{\mathrm{AS}}(t)\right)}$ where the transition matrix $P_{\mathrm{AS}}(t)=\left(\bar{p}_{i j}(t)\right)$ is given as follows: if $\left|\mathcal{N}_{i}(t)\right| \neq 0$, then $\bar{p}_{i j}(t)=1 /\left(N \max \left\{\left|\mathcal{N}_{i}(t)\right|,\left|\mathcal{N}_{j}(t)\right|\right\}\right.$ for $(i, j) \in E(t)$ and $\bar{p}_{i i}(t)=1-\sum_{(i, j) \in E(t)} 1 /\left(N \max \left\{\left|\mathcal{N}_{i}(t)\right|,\left|\mathcal{N}_{j}(t)\right|\right\}\right)$; if $\left|\mathcal{N}_{i}(t)\right|=0$, then $\bar{p}_{i i}(t)=1$. One can easily verify that the matrix $P_{\mathrm{AS}}(t)$ is symmetric and doubly stochastic. The natural random walks $X_{1}$ and $X_{2}$ on the graph $\mathcal{G}(t)$ are equivalent to a single natural random walk, say $X_{M}$, on the product graph $\mathcal{G}(t) \times \mathcal{G}(t)$. That is, $X_{M}$ moving from node $\left(i_{1}, i_{2}\right) \in V \times V$ to node $\left(j_{1}, j_{2}\right) \in V \times V$ on the graph $\mathcal{G}(t) \times \mathcal{G}(t)$ at time $t$, is equivalent to $X_{1}$ moving from $i_{1}$ to $j_{1}$ and $X_{2}$ moving from $i_{2}$ to $j_{2}$ on the graph $\mathcal{G}(t)$ at time $t$. Denote the transition matrix of the random walk $X_{M}$ as $Q(t)=\left(q_{\left(i_{1}, i_{2}\right)\left(j_{1}, j_{2}\right)}(t)\right) \in \mathbb{R}^{N^{2} \times N^{2}}$.

In the following lemma, we will consider the random walk $\bar{X}_{M}$ on the graph $\mathcal{G}(t) \times \mathcal{G}(t)$ with the absorbing set $\Theta$ and the transition matrix $\bar{Q}(t) \in \mathbb{R}^{N^{2} \times N^{2}}$. Denote $e_{\left(\ell_{1}, \ell_{2}\right)}$ by the row corresponding to $\left(\ell_{1}, \ell_{2}\right) \in V \times V$ in a $N^{2} \times N^{2}$ identity matrix. The transition matrix $\bar{Q}(t)$ is defined by replacing the row associated with the absorbing state $\left(\ell_{1}, \ell_{2}\right) \in \Theta$ in $Q(t)$ with $e_{\left(\ell_{1}, \ell_{2}\right)}$. Define $\vartheta_{\left(\ell_{1}, \ell_{2}\right)}(t)=$ $\mathbb{P}\left(X_{M}(t)=\left(\ell_{1}, \ell_{2}\right)\right), \vartheta(t)=\operatorname{col}\left\{\vartheta_{\left(\ell_{1}, \ell_{2}\right)}(t)\right\} \in \mathbb{R}^{N^{2}}, \vartheta_{\Theta}(t)=$ 
$\sum_{\left(\ell_{1}, \ell_{2}\right) \in \Theta} \vartheta_{\left(\ell_{1}, \ell_{2}\right)}(t)$ for the random walk $X_{M}$, and $\bar{\vartheta}_{\left(\ell_{1}, \ell_{2}\right)}(t)=$ $\mathbb{P}\left(\bar{X}_{M}(t)=\left(\ell_{1}, \ell_{2}\right)\right), \bar{\vartheta}(t)=\operatorname{col}\left\{\bar{\vartheta}_{\left(\ell_{1}, \ell_{2}\right)}(t)\right\} \in \mathbb{R}^{N^{2}}, \bar{\vartheta}_{\Theta}(t)=$ $\sum_{\left(\ell_{1}, \ell_{2}\right) \in \Theta} \bar{\vartheta}_{\left(\ell_{1}, \ell_{2}\right)}(t)$ for the random walk $\bar{X}_{M}$.

Lemma 4.1: Consider a network of $N$ nodes whose communication graph $\mathcal{G}(t)$ be undirected and satisfies Assumption 4.1. Let $\left(i_{1}, i_{2}\right) \in$ $V \times V$ be a given node and suppose that the random walks $X_{M}$ and $\bar{X}_{M}$ start from node $\left(i_{1}, i_{2}\right)$ at time 0 . Then it holds that $\bar{\vartheta}_{\Theta}(t) \geq \vartheta_{\Theta}(t) \geq$ $1 /(2 N)$ for $t \geq t_{1}$ where $t_{1}$ is the smallest integer which is larger than $B\left(8 N^{6} \log (\sqrt{2} N)+1\right)$.

Proof: It is not difficult to check that $\mathcal{G}(t) \times \mathcal{G}(t)$ is undirected and satisfies Assumption 4.1 with period $B$. The minimum of nonzero entries in $Q(t)$ is lower bounded by $1 /(N(N-1))$, and $Q(t)$ is symmetric. Observe that for any $\left(i_{1}, i_{2}\right) \in V \times V$ and any $t \geq 0$, $\sum_{\left(j_{1}, j_{2}\right) \in V \times V} q_{\left(i_{1}, i_{2}\right)\left(j_{1}, j_{2}\right)}(t)=\sum_{\left(j_{1}, j_{2}\right) \in V \times V} \bar{p}_{i_{1} j_{1}}(t) \bar{p}_{i_{2} j_{2}}(t)=$ $\sum_{j_{1} \in V} \bar{p}_{i_{1} j_{1}}(t) \times \sum_{j_{2} \in V} \bar{p}_{i_{2} j_{2}}(t)=1$ where we use the fact that the matrix $P_{\mathrm{AS}}(t)$ is doubly stochastic. Hence, the matrix $Q(t)$ is doubly stochastic.

The evolution of $\vartheta(t)$ is governed by the equation $\vartheta(t+1)=$ $Q^{T}(t) \vartheta(t)$ with initial state $\vartheta(0)=e_{\left(i_{1}, i_{2}\right)}^{T}$. Consider the Lyapunov function $V_{1 / N^{2}}(\vartheta)=\sum_{i=1}^{N^{2}}\left(\vartheta_{i}-\left(1 / N^{2}\right)\right)^{2}$ with $V_{1 / N^{2}}(\vartheta(0))=$ $1-\left(1 / N^{2}\right)$. It follows from Lemma 5 in [14] that

$$
V_{\frac{1}{N^{2}}}(\vartheta((k+1) B)) \leq\left(1-\frac{1}{2 N^{5}(N-1)}\right) V_{\frac{1}{N^{2}}}(\vartheta(k B))
$$

for $k \in \mathbb{N}_{0}$. Denote $\mathbf{1} \in \mathbb{R}^{N^{2}}$ as the vector of $N^{2}$ ones and note that

$$
\begin{aligned}
V_{\frac{1}{N^{2}}}(\vartheta(t))-V_{\frac{1}{N^{2}}}(\vartheta(t+1)) & =\left(\vartheta(t)-\frac{1}{N^{2}} \mathbf{1}\right)^{T} \\
\times & \left(I-Q(t) Q^{T}(t)\right)\left(\vartheta(t)-\frac{1}{N^{2}} \mathbf{1}\right) .
\end{aligned}
$$

Since $Q(t)$ is doubly stochastic, so is $Q(t) Q^{T}(t)$. Hence, the diagonal entries of the matrix $\Gamma(t)=I-Q(t) Q^{T}(t)=\left(\gamma_{i j}(t)\right) \epsilon$ $\mathbb{R}^{N^{2} \times N^{2}}$ are dominant in the sense of $\gamma_{i i}(t)=\sum_{j \neq i} \gamma_{i j}(t)$. According to the Gershgorin theorem in [10], all eigenvalues of $\Gamma(t)$ lie in a closed disk centered at $\max _{i \in\left\{1, \cdots, N^{2}\right\}} \gamma_{i i}(t)$ with a radius $\max _{i \in\left\{1, \cdots, N^{2}\right\}} \gamma_{i i}(t)$. Hence, $\Gamma(t)$ is positive semi-definite, and thus $V_{1 / N^{2}}(\vartheta(t))$ is non-increasing along the trajectory of $\vartheta(t)$. Combining (5) with the non-increasing property of $V_{1 / N^{2}}(\vartheta(t))$ gives that

$$
\begin{aligned}
V_{\frac{1}{N^{2}}}(\vartheta(t)) & \leq V_{\frac{1}{N^{2}}}(\vartheta(0))\left(1-\frac{1}{2 N^{5}(N-1)}\right)^{\frac{t}{B}-1} \\
& =\frac{N^{2}-1}{N^{2}}\left(1-\frac{1}{2 N^{5}(N-1)}\right)^{\frac{t}{B}-1} .
\end{aligned}
$$

Since $\vartheta(t)^{T} \mathbf{1}=1$, then $\vartheta_{\min }(t):=\min _{\left(\ell_{1}, \ell_{2}\right) \in V \times V} \vartheta_{\left(\ell_{1}, \ell_{2}\right)}(t) \leq$ $\left(1 / N^{2}\right)$. Since $V_{1 / N^{2}}(\vartheta(t)) \geq\left(\vartheta_{\min }(t)-\left(1 / N^{2}\right)\right)^{2}$, inequality $(6)$ gives that $\vartheta_{\min }(t) \geq \frac{1}{N^{2}}-\left(\frac{N^{2}-1}{N^{2}}\left(1-\frac{1}{2 N^{5}(N-1)}\right)^{(t / B)-1}\right)^{1 / 2}$. Therefore, it holds that $\vartheta_{\min }(t) \geq 1 /\left(2 N^{2}\right)$ for $t \geq B$ $\left(\frac{\left(\log \left(4 N^{2}\left(N^{2}-1\right)\right)\right.}{-\log \left(1-\frac{1}{2 N^{5}(N-1)}\right)}+1\right)$. Since $\log x \leq x-1$, there holds $\left.\left.-1 / \log \left(1-1 /\left(2 N^{5}(N-1)\right)\right)\right)\right) \leq 2 N^{5}(N-1) \leq 2 N^{6}$. Hence, $\vartheta_{\min }(t) \geq 1 /\left(2 N^{2}\right)$ and $\vartheta_{\Theta}(t) \geq 1 /(2 N)$ for $t \geq t_{1}$.

Note that the evolution of $\bar{\vartheta}(t)$ is governed by $\bar{\vartheta}(t+1)=\bar{Q}(t)^{T} \bar{\vartheta}(t)$ with $\bar{\vartheta}(0)=e_{\left(i_{1}, i_{2}\right)}$. Since the set $\Theta$ is absorbing, $\bar{\vartheta}_{\Theta}(t) \geq \vartheta_{\Theta}(t)$ for all $t \geq 0$ and thus the desired result follows.

Proposition 4.1: The meeting time of two natural random walks with transition matrix $P_{\mathrm{AS}}(t)$ on the time-varying graph $\mathcal{G}(t)$ satisfies that $M_{\left(\mathcal{G}(t), P_{\mathrm{AS}}(t)\right)} \leq 4 N t_{1}$.

Proof: Denote by $H_{(\mathcal{G}(t) \times \mathcal{G}(t), Q(t))}(\Theta)$ the hitting time of the random walk $X_{M}$ to reach the set of $\Theta$. Observe that $M_{\left(\mathcal{G}(t), P_{\mathrm{AS}}(t)\right)}=H_{(\mathcal{G}(t) \times \mathcal{G}(t), Q(t))}(\Theta)$. To find an upper bound on $H_{(\mathcal{G}(t) \times \mathcal{G}(t), Q(t))}(\Theta)$, we construct the random walk $X_{M}^{\left(i_{1}, i_{2}\right)}$ in such a way that $X_{M}^{\left(i_{1}, i_{2}\right)}$ starts from $\left(i_{1}, i_{2}\right)$ at time 0 with $i_{1} \neq i_{2}$ and the set $\Theta$ is the absorbing set of $X_{M}^{\left(i_{1}, i_{2}\right)}$. The transition matrix of $X_{M}^{\left(i_{1}, i_{2}\right)}$ is $\bar{Q}(t)$ defined before Lemma 4.1. Define $\vartheta_{\left(\ell_{1}, \ell_{2}\right)}^{\left(i_{1}, i_{2}\right)}(t)=\mathbb{P}\left(X_{M}^{\left(i_{1}, i_{2}\right)}(t)=\left(\ell_{1}, \ell_{2}\right)\right)$, and $\vartheta^{\left(i_{1}, i_{2}\right)}(t)=\operatorname{col}\left\{\vartheta_{\left(\ell_{1}, \ell_{2}\right)}^{\left(i_{1}, i_{2}\right)}(t)\right\} \in \mathbb{R}^{N^{2}}$. The dynamics of $\vartheta^{\left(i_{1}, i_{2}\right)}(t)$ is given by $\vartheta^{\left(i_{1}, i_{2}\right)}(t+1)=\bar{Q}(t)^{T} \vartheta^{\left(i_{1}, i_{2}\right)}(t)$ with the initial state $\vartheta^{\left(i_{1}, i_{2}\right)}(0)=e_{\left(i_{1}, i_{2}\right)}^{T}$.

Define the function $\mu_{\left(\ell_{1}, \ell_{2}\right)}^{\left(i_{1}, i_{2}\right)}: \mathbb{N}_{0} \rightarrow\{0,1\}$ in such a way that $\mu_{\left(\ell_{1}, \ell_{2}\right)}^{\left(i_{1}, i_{2}\right)}=1$ if $X_{M}^{\left(i_{1}, i_{2}\right)}(t)=\left(\ell_{1}, \ell_{2}\right)$; otherwise, $\mu_{\left(\ell_{1}, \ell_{2}\right)}^{\left(i_{1}, i_{2}\right)}(t)=0$ Then, the hitting time $H_{(\mathcal{G}(t) \times \mathcal{G}(t), Q(t), 0)}\left(\left(i_{1}, i_{2}\right), \Theta\right)$ of $X_{M}^{\left(i_{1}, i_{2}\right)}$ equals the expected time that $X_{M}^{\left(i_{1}, i_{2}\right)}$ stays up with the nodes in $V \times V \backslash \Theta$, that is

$$
\begin{aligned}
& H_{(\mathcal{G}(t) \times \mathcal{G}(t), Q(t), 0)}\left(\left(i_{1}, i_{2}\right), \Theta\right) \\
& =\sum_{\left(\ell_{1}, \ell_{2}\right) \notin \Theta} \mathbb{E}\left[\sum_{\tau=0}^{+\infty} \mu_{\left(\ell_{1}, \ell_{2}\right)}^{\left(i_{1}, i_{2}\right)}(\tau)\right] \\
& =\sum_{\tau=0}^{+\infty} \sum_{\left(\ell_{1}, \ell_{2}\right) \notin \Theta} \vartheta_{\left(\ell_{1}, \ell_{2}\right)}^{\left(i_{1}, i_{2}\right)}(\tau) .
\end{aligned}
$$

It follows from Lemma 4.1 that $\vartheta_{\Theta}^{\left(i_{1}, i_{2}\right)}(t) \geq 1 /(2 N)$ for $t \geq t_{1}$. $\vartheta^{\left(i_{1}, i_{2}\right)}(t)^{T} \mathbf{1}=1$ implies that:

$$
\sum_{\left(\ell_{1}, \ell_{2}\right) \notin \Theta} \vartheta_{\left(\ell_{1}, \ell_{2}\right)}^{\left(i_{1}, i_{2}\right)}\left(t_{1}\right) \leq 1-\frac{1}{2 N} .
$$

For each $\left(k_{1}, k_{2}\right) \notin \Theta$, we construct the random walk $\tilde{X}_{M}^{\left(k_{1}, k_{2}\right)}$ in such a way that $\tilde{X}_{M}^{\left(k_{1}, k_{2}\right)}$ starts from $\left(k_{1}, k_{2}\right)$ at time $t_{1}$ and $\Theta$ is the absorbing set of $\tilde{X}_{M}^{\left(k_{1}, k_{2}\right)}$. The transition matrix of $\tilde{X}_{M}^{\left(k_{1}, k_{2}\right)}$ is $\bar{Q}(t)$. Define $\tilde{\vartheta}_{\left(\ell_{1}, \ell_{2}\right)}^{\left(k_{1}, k_{2}\right)}(t)=\mathbb{P}\left(\tilde{X}_{M}^{\left(k_{1}, k_{2}\right)}(t)=\left(\ell_{1}, \ell_{2}\right)\right)$. Following the forgoing arguments for $X_{M}^{\left(i_{1}, i_{2}\right)}$, we have $\sum_{\left(\ell_{1}, \ell_{2}\right) \notin \Theta} \tilde{\vartheta}_{\left(\ell_{1}, \ell_{2}\right)}^{\left(k_{1}, k_{2}\right)}\left(2 t_{1}\right) \leq$ $1-1 /(2 N)$. Combining this with (8) gives that

$$
\begin{aligned}
& \sum_{\left(\ell_{1}, \ell_{2}\right) \notin \Theta} \vartheta_{\left(\ell_{1}, \ell_{2}\right)}^{\left(i_{1}, i_{2}\right)}\left(2 t_{1}\right) \\
& =\sum_{\left(\ell_{1}, \ell_{2}\right) \notin \Theta} \sum_{\left(k_{1}, k_{2}\right) \notin \Theta} \vartheta_{\left(k_{1}, k_{2}\right)}^{\left(i_{1}, i_{2}\right)}\left(t_{1}\right) \tilde{\vartheta}_{\left(\ell_{1}, \ell_{2}\right)}^{\left(k_{1}, k_{2}\right)}\left(2 t_{1}\right) \\
& =\sum_{\left(k_{1}, k_{2}\right) \notin \Theta} \vartheta_{\left(k_{1}, k_{2}\right)}^{\left(i_{1}, i_{2}\right)}\left(t_{1}\right) \sum_{\left(\ell_{1}, \ell_{2}\right) \notin \Theta} \tilde{\vartheta}_{\left(\ell_{1}, \ell_{2}\right)}^{\left(k_{1}, k_{2}\right)}\left(2 t_{1}\right) \\
& \leq\left(1-\frac{1}{2 N}\right)^{2} .
\end{aligned}
$$

By induction, we have $\sum_{\left(\ell_{1}, \ell_{2}\right) \notin \Theta} \vartheta_{\left(\ell_{1}, \ell_{2}\right)}^{\left(i_{1}, i_{2}\right)}\left(n t_{1}\right) \leq$ $(1-1 /(2 N))^{n}$ and then obtain a strictly decreasing sequence $\sum_{\left(\ell_{1}, \ell_{2}\right) \notin \Theta} \vartheta_{\left(\ell_{1}, \ell_{2}\right)}^{\left(i_{1}, i_{2}\right)}\left(n t_{1}\right)$ with respect to $n \in \mathbb{Z}_{0}$. Since the set $\Theta$ is absorbing, then $\sum_{\left(\ell_{1}, \ell_{2}\right) \notin \Theta} \vartheta_{\left(\ell_{1}, \ell_{2}\right)}^{\left(i_{1}, i_{2}\right)}(t)$ is non-increasing with respect to $t \geq 0$. Therefore, we have the following:

$$
\begin{aligned}
\sum_{\left(\ell_{1}, \ell_{2}\right) \notin \Theta} \vartheta_{\left(\ell_{1}, \ell_{2}\right)}^{\left(i_{1}, i_{2}\right)}(t) & \leq \sum_{\left(\ell_{1}, \ell_{2}\right) \notin \Theta} \vartheta_{\left(\ell_{1}, \ell_{2}\right)}^{\left(i_{1}, i_{2}\right)}(0)\left(1-\frac{1}{2 N}\right)^{\frac{t}{t_{1}}-1} \\
& =\left(1-\frac{1}{2 N}\right)^{\frac{t}{t_{1}}-1} .
\end{aligned}
$$

Substituting (10) into (7) gives that

$$
\begin{aligned}
& H_{(\mathcal{G}(t) \times \mathcal{G}(t), Q(t), 0)}\left(\left(i_{1}, i_{2}\right), \Theta\right) \\
& \quad \leq \sum_{\tau=0}^{+\infty}\left(1-\frac{1}{2 N}\right)^{\frac{\tau}{t_{1}}-1} \\
& \quad=\left(1-\frac{1}{2 N}\right)^{-\frac{1}{t_{1}}} \cdot \frac{1}{1-\left(1-\frac{1}{2 N}\right)^{\frac{1}{t_{1}}}} .
\end{aligned}
$$


Since $t_{1}>1$, it holds that $(1-1 /(2 N))^{-\left(1 / t_{1}\right)} \leq 2^{1 / t_{1}}<2$. It follows from Bernoulli's inequality that $(1-1 /(2 N))^{1 / t_{1}} \leq 1-$ $\left(1 / 2 N t_{1}\right)$, and thus $\left(1 /\left(1-(1-1 /(2 N))^{1 / t_{1}}\right)\right) \leq 2 N t_{1}$. Inequality (11) becomes $H_{(\mathcal{G}(t) \times \mathcal{G}(t), Q(t), 0)}\left(\left(i_{1}, i_{2}\right), \Theta\right) \leq 4 N t_{1}$. Actually, this inequality holds for any starting time, any starting node $\left(i_{1}, i_{2}\right)$. Thus it holds that $M_{\left(\mathcal{G}(t), P_{\mathrm{AS}}(t)\right)}=H_{(\mathcal{G}(t) \times \mathcal{G}(t), Q(t),)}(\Theta) \leq 4 N t_{1}$. This completes the proof.

\section{Convergence Analysis of AS}

We are now in the position to characterize the convergence properties of AS. The quantities $T_{\text {con }}(x(0))$ and $T_{\Psi}$ for AS are defined in a similar way to those in Section III.

Theorem 4.1: Let $x(0) \in \mathbb{R}^{N}$ and suppose $x(0) \notin \mathcal{W}(x(0))$. Assume that $\mathcal{G}(t)$ be undirected and satisfies Assumption 4.1. Under AS, almost any evolution $x(t)$ starting from $x(0)$ reaches quantized average consensus. Furthermore, $\mathbb{E}\left[T_{\text {con }}(x(0))\right] \leq$ $(1 / 2) B J(x(0))^{2} N^{2}\left(16 N^{7}+1\right)$.

Proof: The proof is analogous to Theorem 3.1 by using the fact that $\mathbb{E}\left[T_{\Psi}\right]=M_{\left(\mathcal{G}(t), P_{\mathrm{AS}}(t)\right)}$.

\section{DISCUSSION}

Consider a fixed graph $L_{N}^{m}$ with $N$ vertices consists of a clique on $m$ vertices, including vertex $i$, and a path of length $N-m$ with one end connected to one vertex $k \neq i$ of the clique, and the other end of the path being $j$. It was shown in [5] that $H_{\left(L_{N}^{m_{0}}, P_{\mathrm{SF}}\right)}$ is $O\left(N^{3}\right)$ where $m_{0}=\lfloor(2 N+1) / 3\rfloor$. Consider that AF is implemented on the graph $L_{N}^{m_{0}}$ and initial states $x_{i}(0)=0, x_{j}(0)=2$ and $x_{k}(0)=1$ for all $k \neq i, j$. Observe that $\mathbb{E}\left[T_{\text {con }}(x(0))\right]=M_{\left(L_{N}^{m_{0}}, P_{\mathrm{AF}}\right)}$. From Proposition 3.1, we have that $\mathbb{E}\left[T_{\text {con }}(x(0))\right]$ is $O\left(N^{4}\right)$, that is one order less than the bound in Theorem 3.1.

Consider switching graphs $\mathcal{G}(t)$ where $\mathcal{G}(t)$ is the graph $L_{N}^{m_{0}}$ defined above when $t$ is a multiple of $B$; otherwise, all the vertices in $\mathcal{G}(t)$ are isolated. Random walks on $\mathcal{G}(t)$ can be viewed as time-scaled versions of those on $L_{N}^{m_{0}}$, that is, random walks on $\mathcal{G}(t)$ only make the movements when $t$ is a multiple of $B$. Let us consider the case that the algorithm AS is implemented on the graph $L_{N}^{m_{0}}$ and initial states $x_{i}(0)=0, x_{j}(0)=2$ and $x_{k}(0)=1$ for all $k \neq i, j$. Following the same lines above, we have that the bound on $\mathbb{E}\left[T_{\text {con }}(x(0))\right]$ is $O\left(B N^{4}\right)$ which is $N^{4} \log N$-order less than that in Theorem 3.1. One of our future work is to find a tighter upper bound on $\mathcal{G}(t)$.

\section{REFERENCES}

[1] T. C. Aysal, M. J. Coates, and M. Rabbat, "Distributed average consensus using probabilistic quantization," in Proc. IEEE Workshop Stat. Signal Processing, Madison, WI, Aug. 2007, pp. 640-644.

[2] D. P. Bertsekas and J. N. Tsitsiklis, Parallel and Distributed Computation: Numerical Methods. Englewood Cliffs, NJ: Prentice-Hall, 1989.

[3] V. D. Blondel, J. M. Hendrickx, A. Olshevsky, and J. N. Tsitsiklis, "Convergence in multiagent coordination, consensus, and flocking," in Proc. Joint 44th IEEE Conf. Decision Control Eur. Control Conf. (CDC-ECC'05), Seville, Spain, Dec. 2005, pp. 2996-3000.

[4] S. Boyd, A. Ghosh, B. Prabhakar, and D. Shah, "Randomized gossip algorithms," IEEE Trans. Inform. Theory, vol. 52, no. 6, pp. 2508-2530, Jun. 2006

[5] G. Brightwell and P. Winkler, "Maximum hitting time for random walks on graphs," Random Struct. Algorithms, vol. 1, no. 3, pp. 263-276, 1990.

[6] R. Carli, F. Bullo, and S. Zampieri, "Quantized average consensus via dynamic coding/decoding schemes," Int. J. Robust Nonlin. Control, vol. 20, no. 2, pp. 156-175, 2010.

[7] R. Carli, F. Fagnani, P. Frasca, T. Taylor, and S. Zampieri, "Communication constraints in the average consensus problem," Automatica, vol. 44, no. 3, pp. 671-684, 2008.

[8] D. Coppersmith, P. Tetali, and P. Winkler, "Collisions among random walks on a graph," SIAM J. Discrete Math., vol. 6, no. 3, pp. 363-374, 1993.
[9] P. Frasca, R. Carli, F. Fagnani, and S. Zampieri, "Average consensus by gossip algorithms with quantized communication," in Proc. 46th IEEE Conf. Decision Control (CDC'08), Cancun, Mexico, Dec. 2008, pp. 4821-4836.

[10] R. A. Horn and C. R. Johnson, Matrix Analysis. Cambridge, U.K.: Cambridge Univ. Press, 1987.

[11] A. Jadbabaie, J. Lin, and A. S. Morse, "Coordination of groups of mobile autonomous agents using nearest neighbor rules," IEEE Trans. Autom. Control, vol. 48, no. 6, pp. 988-1001, Jun. 2003.

[12] A. Kashyap, T. Basar, and R. Srikant, "Quantized consensus," Automatica, vol. 43, pp. 1192-1203, Jul. 2007.

[13] J. Lavaei and R.M. Murray, "On quantized consensus by means of gossip algorithm,' in Proc. Amer. Control Conf. (ACC'09), St. Louis, MO, Jun. 2009, pp. 394-401.

[14] A. Nedich, A. Olshevsky, A. Ozdaglar, and J. N. Tsitsiklis, "On distributed averaging algorithms and quantization effects," IEEE Trans. Autom. Control, vol. 54, no. 11, pp. 2506-2517, Nov. 2009.

[15] R. Olfati-Saber, J. A. Fax, and R. M. Murray, "Consensus and cooperation in networked multi-agent systems," Proc. IEEE, vol. 95, no. 1, pp. 215-233, Jan. 2007.

[16] A. Olshevsky and J. N. Tsitsiklis, "Convergence speed in distributed consensus and averaging," SIAM J. Control Optim., vol. 48, no. 1, pp. 33-55, 2009.

[17] M. Zhu and S. Martínez, "On the convergence time of distributed quantized averaging algorithms," in Proc. 46th IEEE Conf. Decision Control (CDC'08), Cancun, Mexico, Dec. 2008, pp. 3971-3976.

[18] M. Zhu and S. Martínez, "On the convergence time of asynchronous distributed quantized averaging algorithms," 2010 [Online]. Available: http://arxiv.org/abs/1002.2246

\section{Regulator Synthesis for Bimodal Linear Systems}

Zhizheng Wu, Member, IEEE, and

Foued Ben Amara, Senior Member, IEEE

Abstract-This technical note presents a regulator synthesis approach for switched bimodal linear systems where it is desired to achieve regulation against known disturbance or reference signals. First, a set of observerbased $Q$-parameterized stabilizing controllers for the switched system is constructed. Then regulation conditions for the switched system are obtained and a regulator synthesis method is derived based on iteratively solving a set of linear matrix inequalities. Finally, a numerical example is presented to illustrate the effectiveness of the proposed method.

Index Terms-Bimodal systems, $Q$ parameterization, regulation, switched systems.

\section{INTRODUCTION}

During the last decade, switched systems have attracted considerable attention in the control community [1]-[6]. Such systems are represented by a finite number of subsystems and a rule that governs the switching among these subsystems, resulting in a nonlinear system

Manuscript received July 23, 2009; revised September 12, 2010; accepted September 12, 2010. Date of publication December 06, 2010; date of current version February 09, 2011. This work was supported in part by the Natural Sciences and Engineering Research Council of Canada, the Canada Foundation for Innovation, the Provincial Government of Ontario, and the National Natural Science Foundation of China (51075254). Recommended by Associate Editor C. L. Beck.

Z. Wu is with the Department of Precision Mechanical Engineering, Shanghai University, Shanghai 200072, China (e-mail: zhizhengwu@ shu.edu.cn).

F. Ben Amara is with the Department of Mechanical and Industrial Engineering, The University of Toronto, Toronto, ON M5S 3G8, Canada (e-mail: benamara@mie.utoronto.ca).

Color versions of one or more of the figures in this technical note are available online at http://ieeexplore.ieee.org.

Digital Object Identifier 10.1109/TAC.2010.2090790 